\title{
Clinics in diagnostic imaging (209)
}

Parveen Sulthana Mohamed Ali ${ }^{1}$, MBBS, FRCR, Yet Yen $\underline{Y^{1}}{ }^{1}$, MBBS, FRCR, Tien Jin $\underline{T a n}^{1}$, MBBS, FRCR
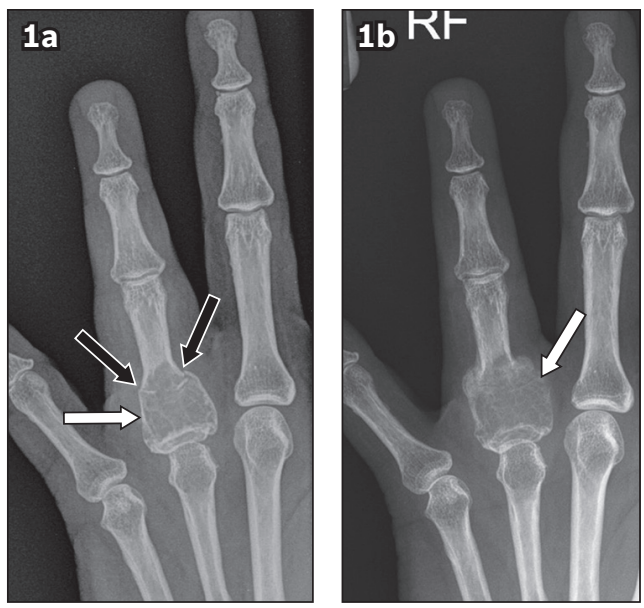

Fig. 1 Posteroanterior radiographs of the left ring finger at (a) the time of presentation and (b) follow-up one month later.
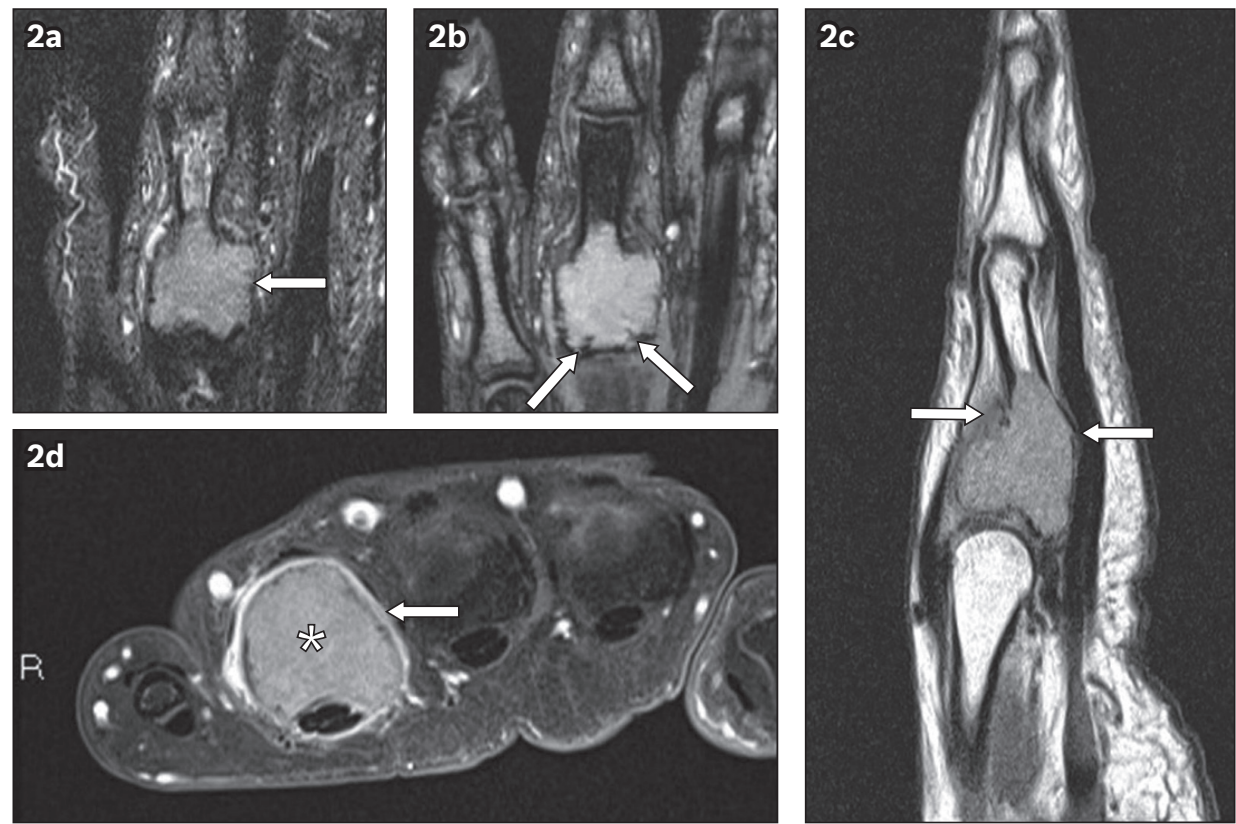

Fig. 2 (a) Coronal turbo inversion recovery magnitude, (b) coronal gradient recovery echo, (c) sagittal proton density, and (d) contrast-enhanced fat-suppressed axial T1-weighted MR images of the left ring finger.

\section{CASE PRESENTATION}

A 53-year-old woman presented to the emergency department with pain and swelling in the left ring finger for three months that had worsened after she accidentally hit her finger against the edge of a table. She had no constitutional symptoms and no significant medical history except for diabetes mellitus. Clinical examination revealed localised swelling and tenderness over the proximal phalanx of the left ring finger. Initial laboratory results showed a total white blood cell count of 8.3 (normal range [NR] 4.0-10.0) $\times 103 / \mathrm{mL}$, C-reactive protein level of $0.4(\mathrm{NR}<3.0 \mathrm{mg} / \mathrm{L})$, serum phosphate level of 1.27 (NR 0.65-1.65 mmol/L) and serum calcium level of 2.34 (NR 2.1-2.6 mmol/L). Plain radiography of the left ring finger was performed at the initial presentation at the emergency department (Fig. 1a) and the one-month follow-up (Fig. 1b). What do the radiographs show? Based on the radiographic findings, magnetic resonance (MR) imaging of the left ring finger (Fig. 2) was subsequently performed. When interpreted with the radiography findings, what is the likely diagnosis? 


\section{IMAGE INTERPRETATION}

The plain radiograph obtained at the initial presentation (Fig. 1a) shows an expansile lytic lesion (white arrow) in the proximal phalanx of the left ring finger associated with an acute, undisplaced, pathological fracture (black arrows). The lesion had a narrow zone of transition, which extended to the subarticular region, and demonstrated thin internal septations giving rise to a 'soap bubble' appearance. No internal chondroid or osteoid matrix mineralisation was seen. There was no significant marginal sclerosis. The two main differential diagnoses based on the radiography findings were enchondroma and giant cell tumour (GCT), with the latter being favoured in view of the patient's age as well as the subarticular location of the lesion.

The follow-up radiograph obtained a month later (Fig. 1b) showed cortical erosion (arrow) and an interval increase in size in keeping with an aggressive bone tumour. MR imaging revealed a solid expansile lytic lesion arising from the left ring finger proximal phalanx, which extended to the proximal subarticular region (Fig. 2). The lesion (arrow, Fig. 2a) demonstrated intermediate signal intensity in the inversion recovery image. Thin linear hypointense internal septations (arrows, Fig. 2b) were also partially seen along the base of the lesion on the gradient recovery echo image but were otherwise better appreciated on radiographs. There was associated cortical thinning and pathological fracture (arrows, Fig. 2c) of the proximal phalanx. There was also avid and fairly homogeneous post-contrast enhancement (asterisk, Fig. 2d) of the lesion (arrow, Fig. 2d).

Overall, the radiograph and MR findings favoured an aggressive primary bone tumour, in particular a GCT of the proximal phalanx. An aggressive chondroid tumour was considered less likely due to the absence of typical chondroid matrix mineralisation on radiographs and lower-than-expected T2-weighted signal return from the lesion on MR imaging.

\section{DIAGNOSIS}

Giant cell tumour of the left ring finger proximal phalanx.

\section{CLINICAL COURSE}

The patient underwent whole-body $99 \mathrm{~m}$-technetium bone scintigraphy and computed tomography (CT) of the chest, abdomen and pelvis. Bone scintigraphy revealed increased radiotracer uptake at the site of the known lesion. There was no synchronous bony lesion or evidence of pulmonary metastasis. The patient was surgically treated with open biopsy, curettage, bone grafting, and partially threaded cancellous screw fixation of the left ring finger proximal phalanx. Biopsy showed lesional tissue composed of sheets of cells, including epithelioid histiocyte-like stromal cells, spindled stromal cells and numerous giant cells compatible with a GCT. Follow-up radiography performed within six months following the initial surgery showed a recurrent lytic lesion with a progressive increase in size and cortical destruction (Fig. 3).

The patient subsequently underwent extensive curettage and bone grafting with $\mathrm{K}$-wire fixation of the left ring finger proximal phalanx. Intraoperative findings showed recurrent GCT involving
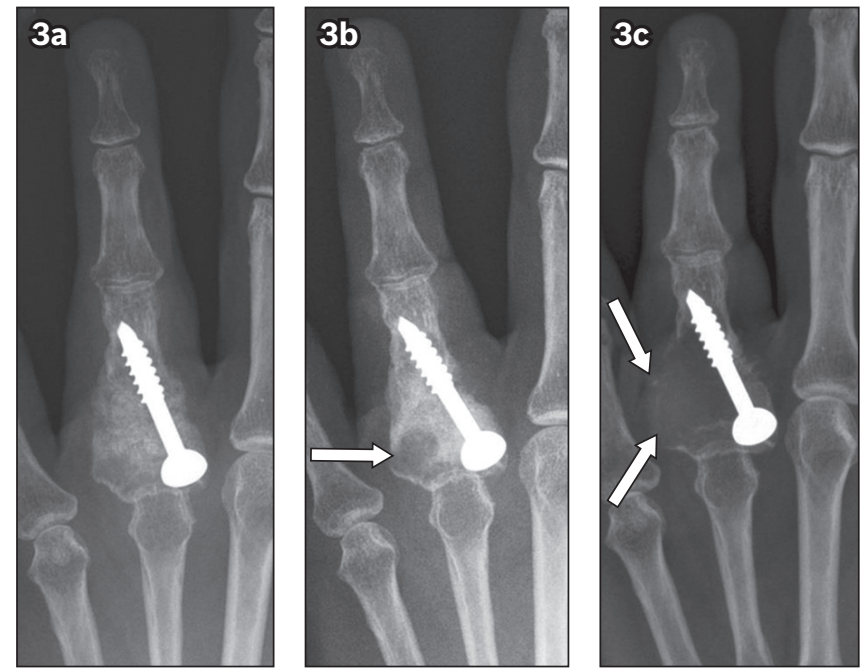

Fig. 3 Follow-up posteroanterior radiographs obtained in the (a) immediate postoperative period following curettage, bone grafting and partially threaded cancellous screw fixation, as well as at (b) three months and (c) six months following the initial surgery show a progressive increase in the size of a lytic lesion at the base of the little finger proximal phalanx with cortical destruction (arrows) suggestive of tumour recurrence.
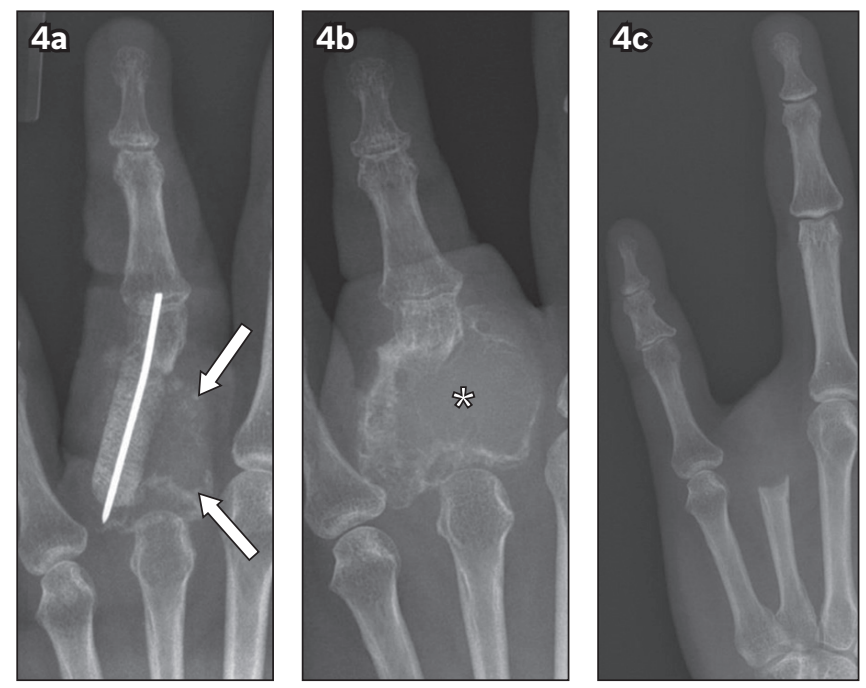

Fig. 4 Follow-up posteroanterior radiographs: (a) following removal of the partially threaded cancellous screw, extensive curettage, bone grafting and K-wire fixation show recurrent lytic lesion (arrows); (b) six months following the removal of the K-wire show an increase in the size of the recurrent lytic lesion (asterisk); and (c) following amputation of the left ring finger.

$>80 \%$ of the native proximal phalanx. Repeated recurrence of the tumour within a year of the second resection was diagnosed on subsequent follow-up radiography, and the patient eventually underwent left ring finger amputation (Fig. 4).

\section{DISCUSSION}

GCT of the bone is a benign but locally aggressive tumour accounting for $5 \%$ of all primary bone tumours and $20 \%$ of benign bone tumours. The tumour is composed of numerous osteoclasttype giant cells seen on a background of uniform mononuclear stromal cells. (1) Previously known as osteoclastoma, the term GCT was first coined by Sir Astley Paston Cooper in 1818 and has remained as the accepted nomenclature. Approximately $80 \%$ of cases occur between the ages of 20 and 50 years, with a slight female predilection. ${ }^{(1)}$ 

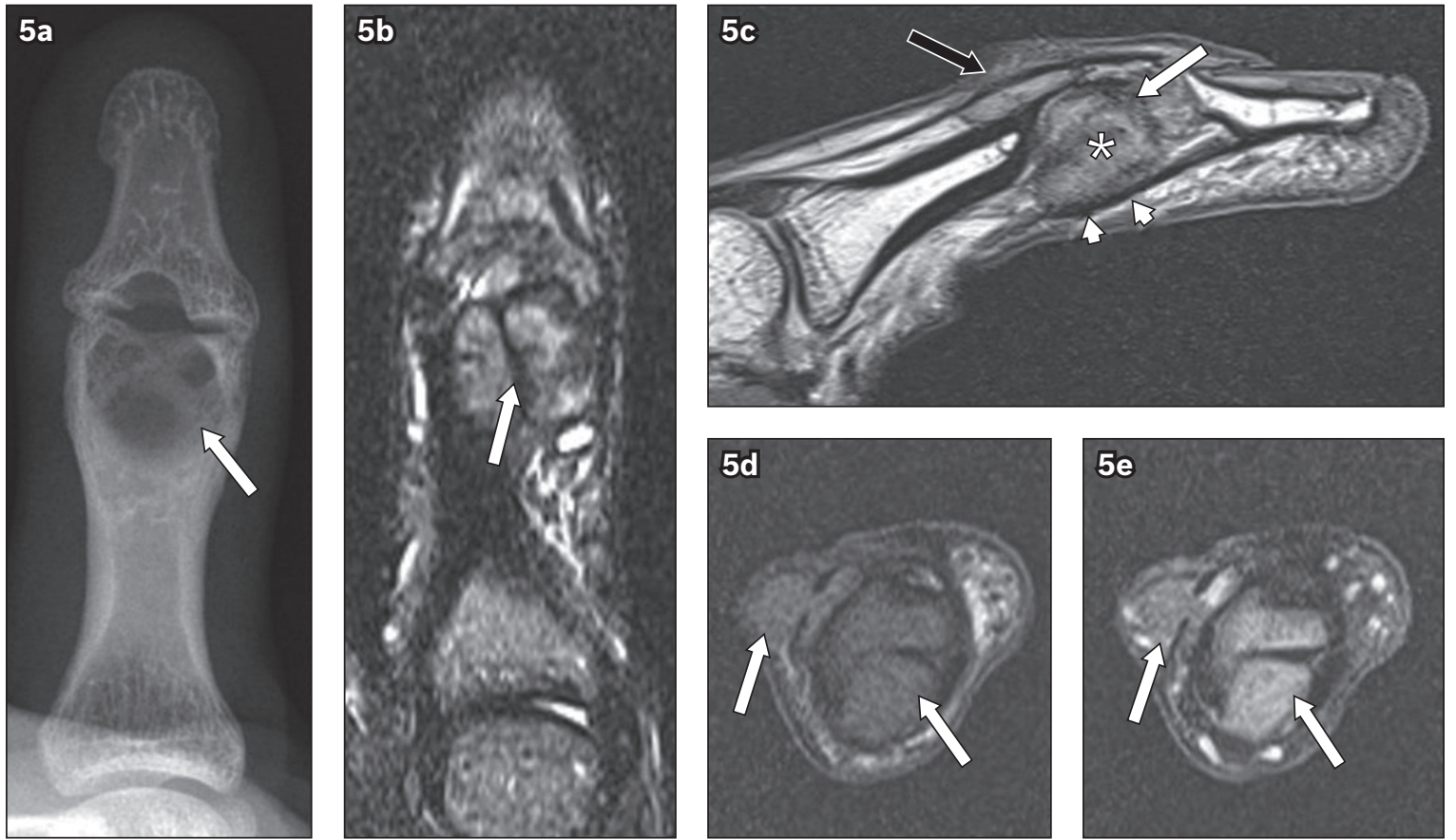

Fig. 5 Giant cell tumour in a 20-year-old man. (a) Posteroanterior radiograph shows a lytic lesion with internal septations in the distal aspect of the thumb proximal phalanx (arrow). (b) Coronal turbo inversion recovery magnitude MR image shows a heterogeneous, intermediate signal intensity appearance of the tumour with thin linear hypointense internal septation (arrow). (c) Sagittal proton density MR image of the tumour (asterisk) shows extraosseous soft tissue extension with involvement of the thumb interphalangeal joint (white arrow), overlying extensor tendon slip (black arrow) and bulging of the underlying flexor pollicis longus tendon (arrowheads). Axial (d) pre- and (e) post-contrast-enhanced T1-weighted MR images show avid post-contrast enhancement of the tumour (arrows).

GCT most commonly occurs around the knee joint, affecting either the distal femur or proximal tibia. The distal radius is the second most common site. In the spine, the sacrum is commonly involved, representing $8 \%$ of cases. Other infrequent sites are flat bones such as the ribs as well as bones of the hands and feet. ${ }^{(1)}$ The tumour usually begins in the metaphysis and extends into the subarticular region. It can be completely metaphyseal in location, although this is rare and is seen in only $1 \%$ of cases.

GCT of the hand is rare, with a reported incidence of approximately $2 \%$. The unique clinical features of this tumour in the hands are that it behaves more aggressively and has a higher rate of tumour recurrence in comparison to GCT at other sites, with reported tumour recurrence usually occurring within a year of the initial diagnosis. ${ }^{(2)}$ Commonly reported sites in the hand include the metacarpals, followed by the phalanges of the digits and, rarely, the thumb (Fig. 5). ${ }^{(3)}$ There is also a slight predilection for younger patients around 20 years of age. ${ }^{(4)}$ Patients usually present with an insidious onset of pain and swelling of their hands, which may be aggravated by a pathological fracture.

The imaging findings of GCT in the hand are similar to those of GCT in the long bones. The typical radiographic features are an expansile, geographic lytic lesion in the subarticular or epimetaphyseal region, associated with cortical thinning or erosion, and non-sclerotic margins. Internal septations within the lesion give rise to the characteristic 'soap bubble' appearance. ${ }^{(1,5)}$ There is typically no significant periosteal reaction. These tumours tend to grow rapidly with invasion into adjacent soft tissues. CT is useful for assessing the tumour margins and the presence of a pathological fracture, which may be occult on radiography. ${ }^{(5)}$ MR imaging is superior in delineating the extent of the tumour as well as in determining the presence of an extraosseous soft tissue component or adjacent joint involvement. The solid component of GCT is usually of intermediate signal intensity on T1-weighted images and of low signal intensity on T2-weighted images due to haemosiderin deposition within the tumour. ${ }^{(6)}$ While not exclusive, the low signal intensity of GCT on T2-weighted images is, however, useful in differentiating this tumour from the majority of other tumours of the digits, which usually show high signal intensity on T2-weighted images. ${ }^{(6)}$ GCT typically demonstrates post-contrast heterogeneous enhancement, although homogeneous enhancement may be observed in smaller lesions. The finding of cystic areas containing fluid-fluid levels in a GCT raises the possibility of secondary aneurysmal bone cyst $(A B C)$ formation. ${ }^{(1,6)}$ Bone scintigraphy usually demonstrates diffusely increased radiotracer uptake by the lesion or peripherally increased radiotracer uptake with central photopenia ('donut' sign). ${ }^{(5)}$

GCT can be multicentric in less than $1 \%$ of patients, and this tends to occur more often in the small bones of the hands and feet. ${ }^{(1)}$ GCT metastases to the lungs are uncommon (1\%-6\% of cases) and have been reported in cases of GCT involving the hand. ${ }^{(1,7)}$ As such, it is imperative that all patients diagnosed with GCT undergo a bone scan and chest CT as part of the diagnostic workup.

Treatment options for GCT of the hand include curettage, curettage with bone grafting, wide resection with reconstruction and amputation. Relatively high recurrence rates have been 
observed following curettage (with or without bone grafting) for GCT of the hand..$^{(8)}$ Denosumab, a chemotherapeutic drug, has shown promising results as an adjuvant treatment to reduce the recurrence rate. ${ }^{(1)}$

The differential diagnoses for a lytic lesion in the small bones of the hand include benign lesions such as enchondroma, $A B C$, brown tumour of hyperparathyroidism and giant cell reparative granuloma. Primary malignant lesions and bony metastases of the hand are infrequently encountered. Other differential diagnoses include infection and crystal deposition disease, such as gout. ${ }^{(9)}$

GCT of the hand can be easily misdiagnosed as enchondroma, which represents the most common benign bone tumour of the hands. Enchondroma is a benign cartilaginous tumour that is typically seen as a centrally placed, well-defined expansile lytic
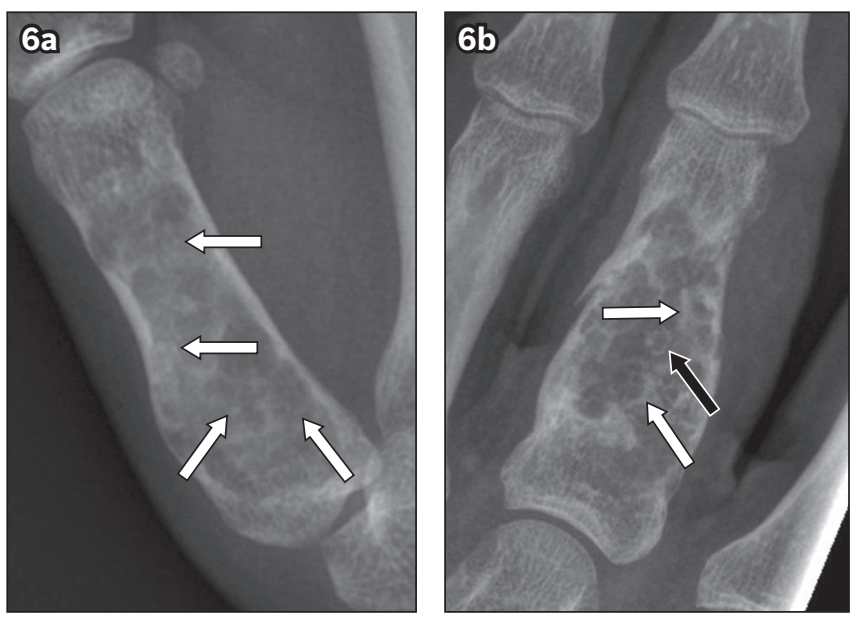

Fig. 6 (a) Frontal radiograph of an enchondroma in the right thumb proximal phalanx of a 24-year-old with 'dot-like' or 'stippled' matrix calcifications (arrows). (b) Frontal radiograph of an enchondroma in the left middle finger proximal phalanx of a 52-year-old man with 'ring' (black arrow) and 'arc' (white arrows) matrix calcifications. lesion with endosteal scalloping or cortical thinning, as well as 'stippled' (dot-like) or 'ring and arc' matrix calcification in the majority of cases (Fig. 6). ${ }^{(10)}$ In lesions without the typical chondroid matrix mineralisation, an enchondroma can mimic other lytic lesions of the tubular bones of the hands (Fig. 7). On MR imaging, an enchondroma demonstrates lobulated margins, high T2-weighted signal intensity due to the high water content of the extracellular matrix, thin internal septa, and low-signal internal foci representing matrix calcifications, and also typically demonstrates peripheral nodular and septal enhancement due to its avascular nature (Fig. 7). ${ }^{(9-11)}$

An $\mathrm{ABC}$ of the hand, a benign but locally aggressive lesion consisting of blood-filled spaces, represents approximately $5 \%$ of benign bone tumours of the hand. These tumours may occur in patients at any age but are typically discovered in patients younger than 30 years of age. ABC usually arises as an expansile lucent lesion in the metaphysis and commonly invades the epiphysis if closure of the physis has occurred. Thin internal septations may be present, giving rise to a 'soap bubble' appearance, but there is no mineralised matrix. MR imaging demonstrates intralesional fluid-fluid levels that are consistent with, but not exclusive to, ABCs. ${ }^{(9,11)}$

A giant cell reparative granuloma (GCRG) is a reactive process of unknown aetiology, although it is postulated to be the sequela of trauma or intratumoral haemorrhage. It is clinically and histologically distinct from GCT. The most common site in the hand for this lesion is the metaphysis or diaphysis of the phalanges. The appearance of GCRG on radiography is an expansile, lytic lesion with expansile remodelling and cortical thinning. Intralesional septation is also typical, but the presence of matrix mineralisation is unusual. ${ }^{(11,12)}$

Chondrosarcoma is the most common primary malignant tumour of the hand. The proximal phalanges of the hand are the preferred sites of occurrence. It usually presents as a
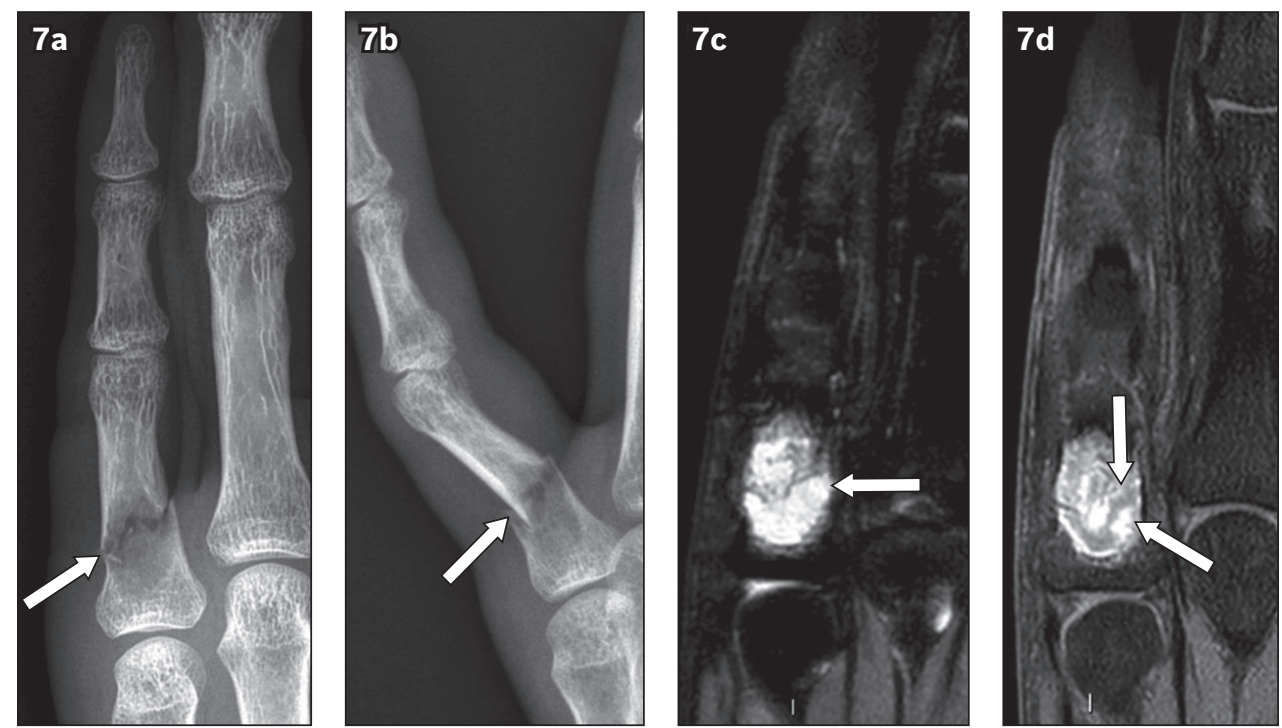

Fig. 7 Enchondroma in a 31-year-old man. (a) Frontal and (b) lateral radiographs show a well-defined lytic lesion in the left little finger proximal phalanx complicated by a pathological fracture (arrows). (c) Coronal turbo inversion recovery magnitude (TIRM) and (d) contrast-enhanced fat-suppressed T1-weighted MR images of the little finger show a marked TIRM (heavily T2-weighted) hyperintense lesion (arrow in Fig. 7c) with peripheral nodular and septal contrast enhancement (arrows in Fig. 7d). 


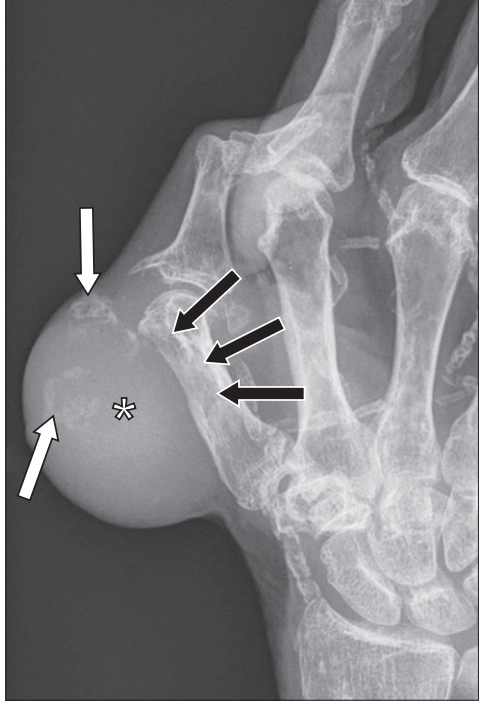

Fig. 8 Chondrosarcoma in an 84-year-old man. Posteroanterior radiograph shows an aggressive osteolytic lesion (black arrows) arising from the first metacarpal, which is associated with a large extraosseous soft tissue component (asterisk) and matrix chondroid calcifications (white arrows).
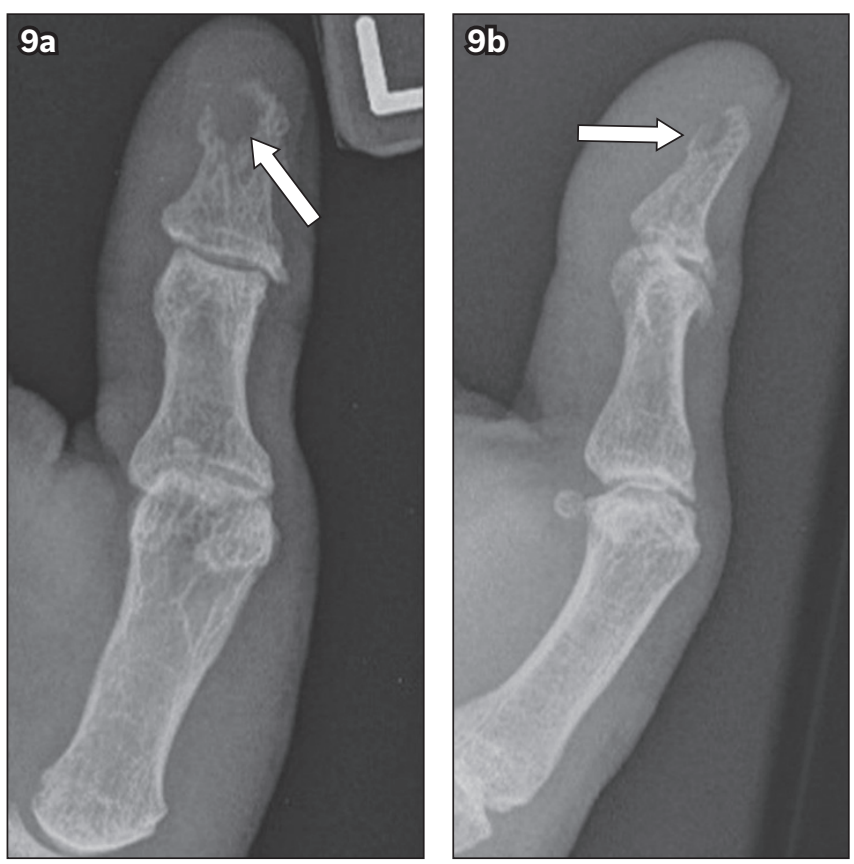

Fig. 9 Bony metastasis in a 61-year-old man with known metastatic bronchogenic carcinoma. (a) Posteroanterior and (b) oblique radiographs of the left thumb show a lytic lesion (arrows) with cortical destruction at the volar aspect of the distal phalangeal tuft. The diagnosis was confirmed histologically.

large, expansile and locally aggressive tumour with cortical destruction, extraosseous soft tissue component and chondroid matrix mineralisation (Fig. 8). It may arise de novo or result from malignant transformation of a pre-existing benign cartilaginous tumour such as an enchondroma. ${ }^{(9-11)}$

Bone metastases to the hands are quite rare and most commonly originate from primary bronchogenic, renal and breast carcinomas (Fig. 9). ${ }^{(9)}$ Systemic disorders such as hyperparathyroidism and gout can have osseous manifestations that can sometimes resemble aggressive primary bone tumours. Appropriate workup of the patient's clinical presentation and
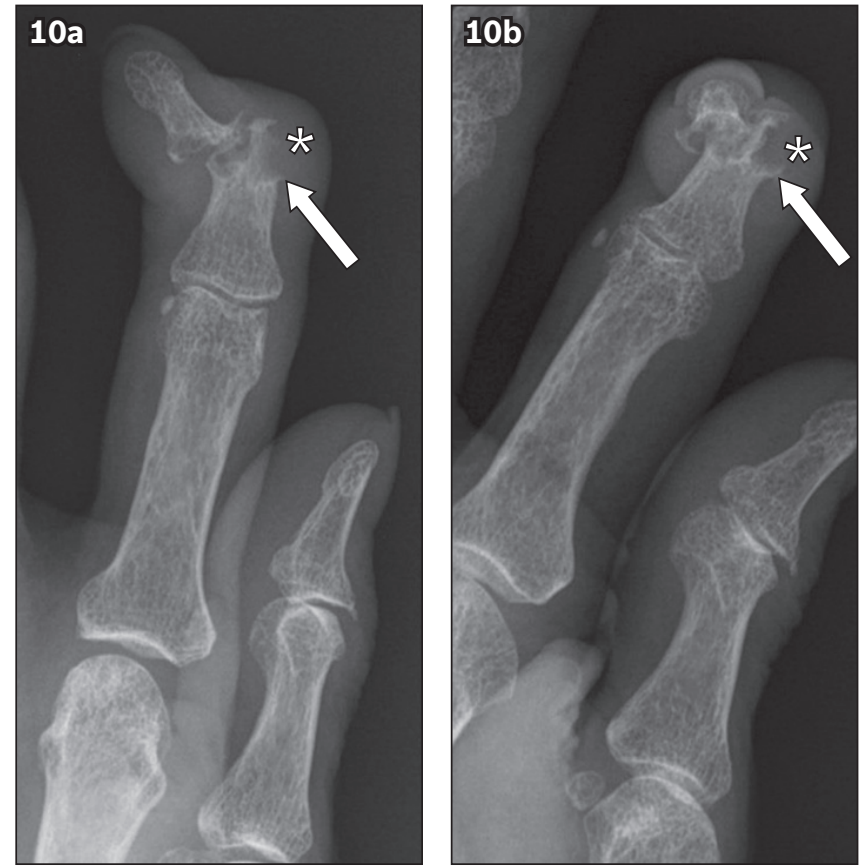

Fig. 10 Chronic tophaceous gout in a 76-year-old woman. (a) Posteroanterior and (b) oblique radiographs of the left index finger show a juxta-articular erosion at the middle phalanx with sclerotic and distinctive overhanging margins (arrows), as well as adjacent soft tissue swelling due to a gouty tophus (asterisks).

biochemistry will be useful if these conditions are suspected clinically.

Brown tumour resulting from hyperparathyroidism, either primary or secondary, occurs due to excessive osteoclastic activity, which results in the formation of destructive bony lesions composed of osteoclasts, multinucleated giant cells and haemorrhage. They appear as expansile, lytic or mixed lyticsclerotic lesions, ${ }^{(13)}$ and if present in the hand, are more commonly seen in the phalanges and may be multiple. The presence of classical radiographic features of hyperparathyroidism in the hand, such as subperiosteal bone resorption along the radial margins of the proximal and middle phalanges of the index and middle fingers, may aid in the diagnosis of these tumours.

Tophaceous gout typically causes well-circumscribed, punched out lytic lesions in the juxta-articular region with classic overhanging bony margins (Fig. 10). ${ }^{(14)}$ There may be associated adjacent soft tissue swelling with or without amorphous calcification secondary to a gouty tophus. Raised serum uric acid levels are usually seen in these patients.

Occasionally, infections can produce aggressive osteolytic lesions in the hand (Fig. 11). The term 'spina ventosa' has been ascribed to expansile, lytic lesions involving the tubular bones of the hand in tuberculous (TB) dactylitis. ${ }^{(15)}$ Cortical thickening and sclerosis may also be present in longstanding cases, while periosteal reaction and sequestra are uncommon. It is noteworthy that approximately $85 \%$ of patients with TB dactylitis are younger than six years of age, with the bones of the hands more frequently affected than bones of the feet. ${ }^{(15)}$

To conclude, GCT of the hands is a rare, benign but locally aggressive tumour with a higher rate of tumour recurrence in 

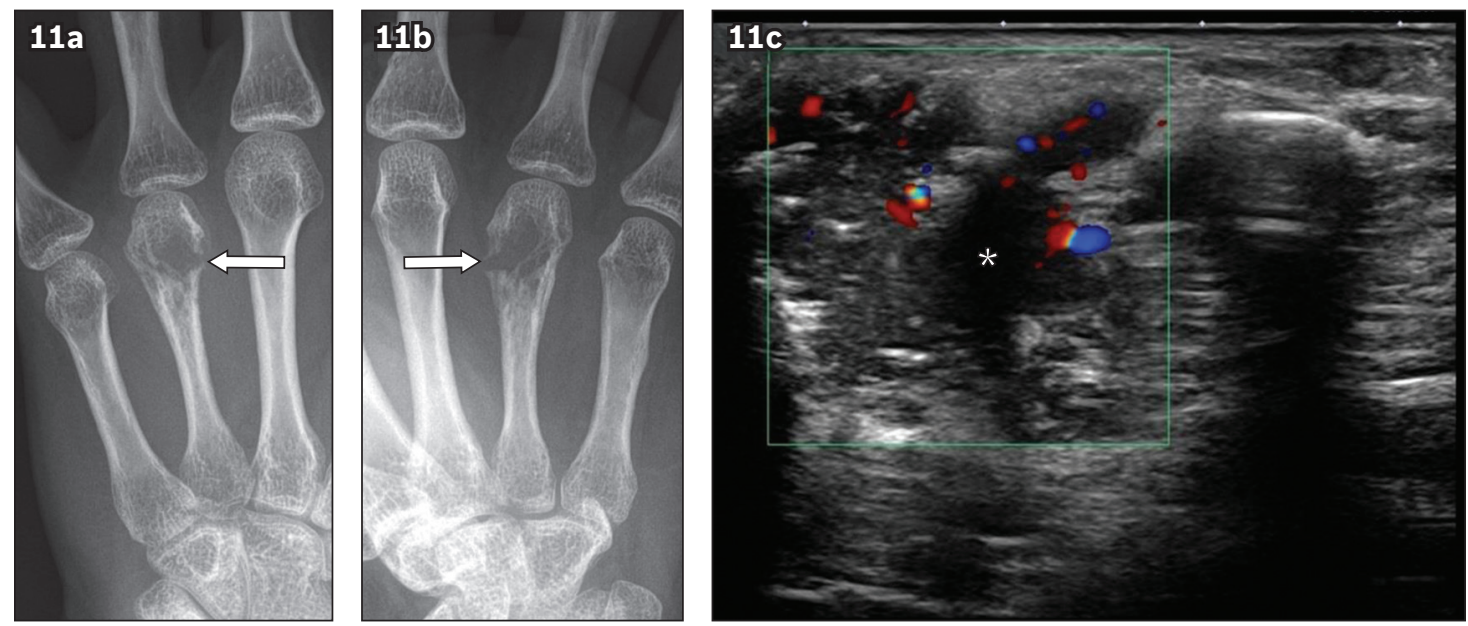

Fig. 11 Tuberculous dactylitis in a 39-year-old woman. Radiographs in the (a) frontal and (b) ball catcher's view of the left middle finger show an ill-defined osteolytic lesion in the fourth metacarpal head and neck with cortical destruction (arrows). (c) Targeted US image of this site in the transverse plane shows a heterogeneous hypoechoic mass with internal vascularity (asterisk). Histological and microbiological analyses were used to confirm the diagnosis.

comparison to GCT at other sites. Imaging plays an essential role in the accurate diagnosis and management of this tumour. Knowledge of the differential diagnoses of GCT of the hand would be useful to the reporting radiologist and referring clinician in the diagnostic workup of such patients.

\section{REFERENCES}

1. Chakarun CJ, Forrester DM, Gottsegen CJ, et al. Giant cell tumor of bone: review, mimics, and new developments in treatment. Radiographics 2013; 33:197-211.

2. Meena UK, Sharma YK, Saini N, Meena DS, Gahlot N. Giant cell tumours of hand bones: a report of two cases. J Hand Microsurg 2015; 7:177-81.

3. Reichert P, Kowalski P, Gosk J. The giant cell tumour of the proximal phalanx of the thumb treated by a 2-stage operation. Acta Orthop Traumatol Turc 2017; 51:425-8

4. Biscaglia R, Bacchini P, Bertoni F. Giant cell tumor of the bones of the hand and foot. Cancer 2000; 88:2022-32.

5. James SLJ, Davies AM. Giant-cell tumours of bone of the hand and wrist: a review of imaging findings and differential diagnoses. Eur Radiol 2005; 9:1855-66.

6. Pereira HM, Marchiori E, Severo A. Magnetic resonance imaging aspects of giant-cell tumours of bone. J Med Imaging Radiat Oncol 2014; 58:674-8.

7. Jacopin S, Viehweger $E$, Glard $Y$, et al. Fatal lung metastasis secondary to index finger giant cell tumour in an 8-year-old child. Orthop Traumatol Surg Res 2010; 96:310-3.

8. Adulkasem N, Pruksakorn D. Giant cell tumour of the middle phalanx of the middle finger. BMJ Case Rep 2019; 12:e229076.

9. Melamud K, Drapé JL, Hayashi D, et al. Diagnostic imaging of benign and malignant osseous tumours of the fingers. Radiographics 2014; 34:1954-67.

10. Khoo RN, Peh WC, Guglielmi G. Clinics in diagnostic imaging (124). Multiple enchondromatosis in Ollier disease. Singapore Med J 2008; 49:841-5.

11. Ahmed O, Moore DD, Stacy GS. Imaging diagnosis of solitary tumors of the phalanges and metacarpals of the hand. AJR Am J Roentgenol 2015; 205:106-15

12. Murphey MD, Nomikos GC, Flemming DJ, et al. From the archives of AFIP. Imaging of giant cell tumor and giant cell reparative granuloma of bone: radiologic-pathologic correlation. Radiographics 2001; 21:1283-309.

13. Plate AM, Lee SJ, Steiner G, Posner MA. Tumorlike lesions and benign tumors of the hand and wrist. J Am Acad Orthop Surg 2003; 11:129-41.

14. Yochum TR, Rowe LJ. Essentials of skeletal radiology. 3rd ed. Vol 2. Philadelphia: Lippincott Williams and Wilkins, 2005

15. Andronikou S, Smith B. "Spina ventosa"--tuberculous dactylitis. Arch Dis Child 2002; 86:206. 


\section{SINGAPORE MEDICAL COUNCIL CATEGORY 3B CME PROGRAMME} (Code SMJ 202102B)

Question 1. The most common site of giant cell tumour (GCT) of the bone is:

(a) Proximal humerus.

(b) Around the knee involving either the distal femur or proximal tibia.

(c) Metacarpal.

(d) Rib.

Question 2. The unique clinical features of GCT involving the bones of the hand, when compared to other locations in the axial or appendicular skeleton, include:
(a) Non-aggressive nature.
(b) Predilection for younger patients around 20 years of age.
(c) Higher rate of recurrence.
(d) Occurs exclusively in older patients.

Question 3. Imaging features of a GCT of the bone include:
(a) Rapid growth.
(b) Expansile nature.
(c) Soap bubble appearance.
(d) Cortical erosion.

Question 4. Low signal intensity seen within a GCT of the bone on T2-weighted magnetic resonance imaging is typically due to:
(a) Calcification.
(b) Haemosiderin.
(c) Water.
(d) Fat.

Question 5. 'Stippled' (dot-like) or 'ring and arc' type of matrix mineralisation in a lytic bone lesion of the hand is characteristic of:
(a) GCT.
(b) Enchondroma
(c) Aneurysmal bone cyst.
(d) Giant cell reparative granuloma.

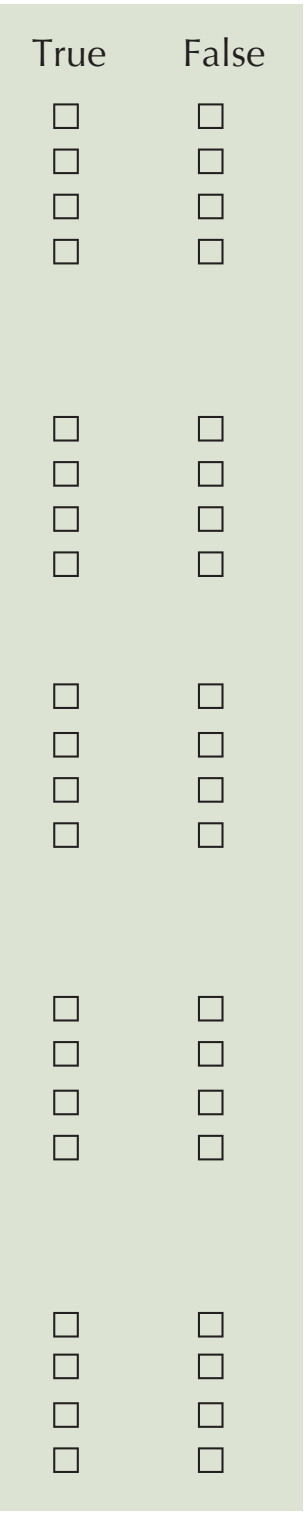

\section{Doctor's particulars:}

Name in full:

MCR no.:

Specialty:

Email:

\section{SUBMISSION INSTRUCTIONS:}

Visit the SMJ website: http://www.smj.org.sg/current-issue and select the appropriate quiz. You will be redirected to the SMA login page.

For SMA member: (1) Log in with your username and password (if you do not know your password, please click on 'Forgot your password?'). (2) Select your answers for each quiz and click 'Submit'.

For non-SMA member: (1) Create an SMJ CME account, or log in with your SMJ CME username and password (for returning users). (2) Make payment of SGD 21.40 (inclusive of $7 \%$ GST) via PayPal to access this month's quizzes. (3) Select your answers for each quiz and click 'Submit'.

RESULTS:

(1) Answers will be published online in the SMJ April 2021 issue. (2) The MCR numbers of successful candidates will be posted online at the SMJ website by 10 April 2021. (3) Passing mark is $60 \%$. No mark will be deducted for incorrect answers. (4) The SMJ editorial office will submit the list of successful candidates to the Singapore Medical Council. (5) One CME point is awarded for successful candidates. (6) SMC credits CME points according to the month of publication of the CME article (i.e. points awarded for a quiz published in the February 2021 issue will be credited for the month of February 2021, even if the deadline is in April 2021).

Deadline for submission (February 2021 SMJ 3B CME programme): 12 noon, 3 April 2021. 